\title{
Bernoulli Processes in Riesz spaces *
}

\author{
Wen-Chi Kuo ${ }^{\dagger}$ \\ School of Computational and Applied Mathematics \\ University of the Witwatersrand \\ Private Bag 3, P O WITS 2050, South Africa
}

\author{
Jessica Vardy \& Bruce A. Watson $^{\S}$ \\ School of Mathematics \\ University of the Witwatersrand \\ Private Bag 3, P O WITS 2050, South Africa
}

September 21, 2018

\begin{abstract}
The action and averaging properties of conditional expectation operators are studied in the, measure-free, Riesz space, setting of Kuo, Labuschagne and Watson [ Conditional expectations on Riesz spaces, J. Math. Anal. Appl., 303 (2005), 509-521] but on the abstract $L^{2}$ space, $\mathcal{L}^{2}(T)$ introduced by Labuschagne and Watson [ Discrete Stochastic Integration in Riesz Spaces, Positivity, 14, (2010), 859 - 575]. In this setting it is shown that conditional expectation operators leave $\mathcal{L}^{2}(T)$ invariant and the Bienaymé equality and Tchebichev inequality are proved. From this foundation Bernoulli processes are considered. Bernoulli's strong law of large numbers and Poisson's theorem are formulated and proved.
\end{abstract}

\section{Introduction}

Various authors have considered generalizations of stochastic processes to vector lattices / Riesz spaces, with a variety of assumptions being made on the processes being considered. Most of this work has focussed on martingale theory, see, for example, [4], [7, 10],

*Keywords: Riesz spaces, Vector lattices, conditional expectation operators, $f$-algebra, averaging operators, Bernoulli processes, conditional independence, strong laws of large numbers, Mathematics subject classification (2010): 47B60; 47B80; 60B12.

${ }^{\dagger}$ Research conducted while on an NRF post doctoral fellowship

${ }^{\ddagger}$ Funded in part by the FRC

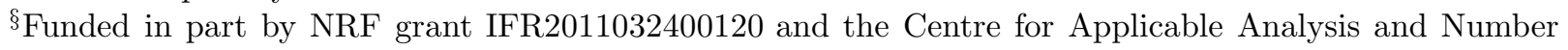
Theory 
[9], [16] and [17]. The abstract properties of conditional expectation operators have also been explored in various settings, see [5], 11], [14] and [15] and [19]. However, the more elementary processes such as Markov processes, see [18], Bernoulli processes and Poisson processes, which just rely only on the concepts of a conditional expecation operator and independence, have received little attention. As these processes have less accessible structure, their study relies more heavily on properties of the underlying Riesz space, the representation of the conditional expectation operators and multiplication operations in Riesz spaces. If a Riesz space has a weak order unit, then the order ideal generated by a weak order unit is order dense in the space. However the order ideal generated by the weak order unit is an $f$-algebra, see [2], 3] and [20], giving a multiplicative structure on a dense subspace. Much of the work in this paper lies on a Riesz space vector analogue of $L^{2}$ and the action of conditional expectations in this space, see Theorem $[3.2$, and their averaging property, see Lemma 3.1. In particular the Bienaymé equality, Theorem 4.2, will be posed in this setting. The Bienaymé equality enables us to give a Riesz space analogue of Bernoulli's law of large numbers, Theorem 5.2. One important property of the ideal generated by the weak order unit is that it posesses a functional calculus which enables one to lift continuous real valued functions on $[0,1]$ to the Riesz space, see [6] and [20]. This is critical for Poisson's theorem, Theorem [5.6. We refer the reader to [13] for the classical version of the Bienaymé equality, the Bernoulli law of large numbers and Poisson's theorem.

\section{$2 \quad$ Riesz Space Preliminaries}

We refer the reader to [1] and 20] for general Riesz space theory. The definitions and preliminaries presented here are specific to Riesz spaces with a weak order unit and a conditional expectation operator.

The notion of a conditional expectation operator in a Dedekind complete Riesz space, $E$, with weak order unit was introduced in [10] as a positive order continuous projection $T: E \rightarrow E$, with range $\mathcal{R}(T)$ a Dedekind complete Riesz subspace of $E$, and having $T e$ a weak order unit of $E$ for each weak order unit $e$ of $E$. Instead of requiring $T e$ to be a weak order unit of $E$ for each weak order unit $e$ of $E$ one can equivalently impose that there is a weak order unit in $E$ which is invariant under $T$. Averaging properties of conditional expectation operators and various other structural aspects were considered in [11]. In particular if $B$ is the band in $E$ generated by $0 \leq g \in \mathcal{R}(T)$ and $P$ is the band projection onto $B$, it was shown that $T f \in B$, for each $f \in B, P f,(I-P) f \in \mathcal{R}(T)$ for each $f \in \mathcal{R}(T)$, where $I$ denotes the identity map, and $T f \in B^{d}$, for each $f \in B^{d}$. A consequence of these relations and Freudenthal's theorem, [20], is that if $B$ is the band in $E$ generated by $0 \leq g \in \mathcal{R}(T)$, with associated band projection $P$, then $T P=P T$, see [11] for details.

To access the averaging properties of conditional expectation operators a multiplicative structure is needed. In the Riesz space setting the most natural multiplicative structure is that of an $f$-algebra. This gives a multiplicative structure that is compatible with the order and additive structures on the space. The ideal, $E^{e}$, of $E$ generated by $e$, 
where $e$ is a weak order unit of $E$ and $E$ is Dedekind complete, has a natural $f$-algebra structure. This is constructed by setting $(P e) \cdot(Q e)=P Q e=(Q e) \cdot(P e)$ for band projections $P$ and $Q$, and extending to $E^{e}$ by use of Freudenthal's Theorem. In fact this process extends the multiplicative structure to the universal completion, $E^{u}$, of $E$. This multiplication is associative, distributive and is positive in the sense that if $x, y \in E^{+}$ then $x y \geq 0$. Here $e$ is the multiplicative unit. For more information about $f$-algebras see [2, 3, 5, 8, 11, 20]. If $T$ is a conditional expectation operator on the Dedekind complete Riesz space $E$ with weak order unit $e=T e$, then restricting our attention to the $f$-algebra $E^{e}, T$ is an averaging operator on $E^{e}$ if $T(f g)=f T g$ for $f, g \in E^{e}$ and $f \in R(T)$, see [5, 8, 11]. More will said about averaging operators in Section 3 .

In a Dedekind complete Riesz space, $E$, with weak order unit and $T$ a strictly positive conditional expectation on $E$. We say that the space is $T$-universally complete if for each increasing net $\left(f_{\alpha}\right)$ in $E_{+}$with $\left(T f_{\alpha}\right)$ order bounded in the universal completion $E^{u}$, we have that $\left(f_{\alpha}\right)$ is order convergent. If this is not the case, then both the space and conditional expectation operator can be extended so that the extended space is $T$-universally complete with respect to the extended $T$, see [11. The extended space is also know as the natural domain of $T$, denoted $\operatorname{dom}(T)$ of $\mathcal{L}^{1}(T)$, see [5, 8].

Let $E$ be a Dedekind complete Riesz space with conditional expectation $T$ and weak order unit $e=T e$. If $P$ and $Q$ are band projections on $E$, we say that $P$ and $Q$ are $T$-conditionally independent with respect to $T$ if

$$
T P T Q e=T P Q e=T Q T P e .
$$

We say that two Riesz subspaces $E_{1}$ and $E_{2}$ of $E$ are $T$-conditionally independent with respect to $T$ if all band projections $P_{i}, i=1,2$, in $E$ with $P_{i} e \in E_{i}, i=1,2$, are $T$ conditionally independent with respect to $T$. Equivalently (2.1) can be replaced with

$$
T P T Q w=T P Q w=T Q T P w \quad \text { for all } \quad w \in \mathcal{R}(T) .
$$

It should be noted that $T$-conditional independence of the band projections $P$ and $Q$ is equivalent to $T$-conditional independence of the closed Riesz subspaces $\langle P e, \mathcal{R}(T)\rangle$ and $\langle Q e, \mathcal{R}(T)\rangle$ generated by $P e$ and $\mathcal{R}(T)$ and by $Q e$ and $\mathcal{R}(T)$ respectively. From the Radon-Nikodým-Douglas-Andô type theorem was established in [19], if $E$ is a $T$ universally complete, a subset $F$ of $E$ is a closed Riesz subspace of $E$ with $\mathcal{R}(T) \subset F$ if and only if there is a unique conditional expectation $T_{F}$ on $E$ with $\mathcal{R}\left(T_{F}\right)=F$ and $T T_{F}=T=T_{F} T$. In this case $T_{F} f$ for $f \in E^{+}$is uniquely determined by the property that

$$
T P f=T P T_{F} f
$$

for all band projections on $E$ with $P e \in F$. As a consequence of this, two closed Riesz subspaces $E_{1}$ and $E_{2}$ with $\mathcal{R}(T) \subset E_{1} \cap E_{2}$ are $T$-conditionally independent, if and only if

$$
T_{1} T_{2}=T=T_{2} T_{1}
$$

where $T_{i}$ is the conditional expectation commuting with $T$ and having range $E_{i}, i=1,2$. Here (2.4) can be equivalently replaced by

$$
T_{i} f=T f, \quad \text { for all } f \in E_{3-i}, \quad i=1,2,
$$


see [18]. The concept of $T$-conditional independence can be extended to a family, say $\left(E_{\lambda}\right)_{\lambda \in \Lambda}$, of closed Dedekind complete Riesz subspaces of $E$ with $\mathcal{R}(T) \subset E_{\lambda}$ for all $\lambda \in \Lambda$. We say that the family is $T$-conditionally independent if, for each pair of disjoint sets $\Lambda_{1}, \Lambda_{2} \subset \Lambda$, we have that $E_{\Lambda_{1}}$ and $E_{\Lambda_{2}}$ are $T$-conditionally independent. Here $E_{\Lambda_{j}}:=\left\langle\bigcup_{\lambda \in \Lambda_{j}} E_{\lambda}\right\rangle$. Finally, we say that a sequence $\left(f_{n}\right)$ in $E$ is $T$-conditionally independent if the family of closed Riesz subspaces $\left\langle\left\{f_{n}\right\} \cup \mathcal{R}(T)\right\rangle, n \in \mathbb{N}$, is $T$-conditionally independent.

\section{Conditional expectation operators in $\mathcal{L}^{2}(T)$}

In this work we assume that $E$ is $T$-universally complete and in this case we have $\mathcal{L}^{1}(T)=E$, see [9]. As $E^{u}$, the universal completion of $E$, is an $f$-algebra, multiplication of elements of $E$ is defined but does not necessarily result in an element of $E$. This leads us, as in [9], to define

$$
\mathcal{L}^{2}(T):=\left\{x \in \mathcal{L}^{1}(T) \mid x^{2} \in \mathcal{L}^{1}(T)\right\} .
$$

If $f, g \in \mathcal{L}^{2}(T)$ then in the $f$-algebra $E^{u}, 0 \leq(f \pm g)^{2}=f^{2} \pm 2 f g+g^{2}$. Thus $\pm 2 f g \leq$ $f^{2}+g^{2}$ and $2|f g| \leq f^{2}+g^{2} \in \mathcal{L}^{1}(T)=E$. Hence $f g \in \mathcal{L}^{1}(T)=E$. As noted in [9], a consequence of this is that $\mathcal{L}^{2}(T)$ is a vector space.

The averaging property of conditional expectation operators only makes sense if it can be ensured that the all products involved remain in the space. Theorem 4.3 of [11] states that if $E$ is a Dedekind complete Riesz space with weak order unit, $T$ is a conditional expectation operator on $E$ and $E$ is also an $f$-algebra, then $T$ is an averaging operator, i.e. $T(f g)=g T f$ for all $f \in E, g \in \mathcal{R}(T)$. The averaging property is revisited in [9, Theorem 2.1] without proof. The variant of [9, Theorem 2.1] drops the assumption that $E$ is an $f$-algebra, but imposes the additional conditions that $f g \in E$ and that $E$ is $T$-universally complete. A strengthened version of this is proved in Lemma 3.1. This however does not address whether $S f \in \mathcal{L}^{2}(T)$ for $f \in \mathcal{L}^{2}(T)$ and $S$ a conditional expectation operator on $E$ with $T S=T=S T$. For this see Theorem 3.2 below. As a consequence of Lemma 3.1 and Theorem 3.2 , we are able to conclude, see Theorem 3.3 below, that for such a conditional expectation operator, $S, S(f T g)=T g \cdot S f$ for all $f, g \in \mathcal{L}^{2}(T)$.

Lemma 3.1 Let $E$ be a Dedekind complete Riesz space with weak order unit, e, and $T$ is a conditional expectation operator on $E$ with $T e=e$. If $f, g, f g \in E$ with $g \in \mathcal{R}(T)$ then $g \cdot T f \in E$ and $T(f g)=g \cdot T f$.

Proof: Case I: $f, g, f g \in E_{+}$with $g \in \mathcal{R}(T)$ Let $f_{n}=f \wedge n e$ and $g_{n}=g \wedge n e$. Then $f_{n} \uparrow f \overline{\text { and } g_{n} \uparrow g \text {. Here } f_{n}, g_{n} \in E_{+}^{e} \text { with }} g_{n} \in \mathcal{R}(T)$, so [11, Theorem 4.3] can be applied to give $T\left(f_{n} g_{m}\right)=g_{m} T\left(f_{n}\right), m, n \in \mathbb{N}$. Thus

$$
g_{m} T\left(f_{n}\right)=T\left(f_{n} g_{m}\right) \leq T(f g), \quad m, n \in \mathbb{N} .
$$


Here $f_{n} g_{m} \uparrow f g$ in $E$, so from the order continuity of $T, T\left(f_{n} g_{m}\right) \uparrow T(f g)$ in $E$. In the universal completion, $E^{u}$, of $E$, we have $g_{m} T\left(f_{n}\right) \uparrow g T(f)$, however, from (3.1), $g_{m} T\left(f_{n}\right)$ is bounded above by $T(f g) \in E$, so $g_{m} T\left(f_{n}\right) \uparrow g T(f)$ in $E$, giving $T(f g)=g T(f)$.

Case II: $f, g, f g \in E$ with $g \in \mathcal{R}(T)$ From Case I, $T\left(f^{ \pm} g^{\mp}\right)=g^{\mp} T\left(f^{ \pm}\right)$and $T\left(f^{ \pm} g^{ \pm}\right)=$ $\overline{g^{ \pm} T\left(f^{ \pm}\right) \text {, from which the result follows. }}$

Theorem 3.2 Let E be a T-universally complete Riesz space with weak order unit, e, where $T$ is a strictly positive conditional expectation operator with $T e=e$ and let $S$ be a conditional expectation operator on $E$ with $T S=T=S T$. If $f \in \mathcal{L}^{2}(T)$ then $S f \in \mathcal{L}^{2}(T)$.

Proof: Let $f \in \mathcal{L}^{2}(T)$ and define $f_{n}=(n e \wedge|f|) \in E_{+}^{e}, n \in \mathbb{N}$. We note that $E^{e}$ is an $f$-algebra. Here $S f_{n} \in E_{+}^{e}$ and $f_{n}-S f_{n} \in E^{e}$ and so $\left(f_{n}-S f_{n}\right)^{2} \in E_{+}^{e}$. But

$$
\left(f_{n}-S f_{n}\right)^{2}=f_{n}^{2}-2 f_{n} \cdot S f_{n}+\left(S f_{n}\right)^{2} .
$$

Thus,

$$
0 \leq S\left(f_{n}-S f_{n}\right)^{2}=S f_{n}^{2}-2 S\left(f_{n} \cdot S f_{n}\right)+S\left(S f_{n}\right)^{2} .
$$

As conditional expectation operators on $E^{e}$ are averaging operators, see [11, for each $g \in E^{e}$ we have

$$
S\left(g \cdot S f_{n}\right)=S f_{n} \cdot S g
$$

Taking $g=f_{n}$ in (3.3) gives

$$
S\left(f_{n} \cdot S f_{n}\right)=S f_{n} \cdot S f_{n}=\left(S f_{n}\right)^{2},
$$

while taking $g=S f_{n}$ gives

$$
S\left(S f_{n} \cdot S f_{n}\right)=S f_{n} \cdot S\left(S f_{n}\right)=\left(S f_{n}\right)^{2},
$$

as $S$ is a projection. Combining (3.4) and (3.5) with (3.2) gives

$$
S f_{n}^{2} \geq\left(S f_{n}\right)^{2} .
$$

Since $f_{n} \uparrow|f|$ we have $f_{n}^{2} \uparrow|f|^{2}$ and $S f_{n}^{2} \uparrow S|f|^{2}$, as $n \rightarrow \infty$, from the order continuity of $S$. Similarly $S f_{n} \uparrow S|f|$ giving $\left(S f_{n}\right)^{2} \uparrow(S|f|)^{2}$ as $n \rightarrow \infty$. Hence taking $n \rightarrow \infty$ in (3.6) yields

$$
(S|f|)^{2} \leq S f^{2}
$$

But $|S f| \leq S|f|$ so

$$
(S f)^{2} \leq S f^{2} \in E
$$

giving $S f \in \mathcal{L}^{2}(T)$.

Corollary 3.3 Let E be a T-universally complete Riesz space with weak order unit, e, where $T$ is a strictly positive conditional expectation operator with $T e=e$. Let $S, J$ be conditional expectation operators on $E$ with $T S=T=S T, T J=T=J T$ and $J S=J=S J$. If $f, g \in \mathcal{L}^{2}(T)$ then $S(f \cdot J g)=J g \cdot S(f)$. 
Proof: As $f, g \in \mathcal{L}^{2}(T)$ from Theorem $3.2 J g \in \mathcal{L}^{2}(T)$. Now $f, J g \in \mathcal{L}^{2}(T)$ so $f, J g, f$. $J g \in E$ so Lemma 3.1 gives $J g \cdot S f \in E$ and $S(f \cdot J g)=J g \cdot S f$.

We are now in a position to give the Tchebichev's inequality in $\mathcal{L}^{2}(T)$.

Theorem 3.4 (Tchebichev's Inequality) Let $E$ be a Dedekind complete Riesz space with conditional expectation $T$ and weak order unit $e=T e$. Let $f \in \mathcal{L}^{2}(T), f \geq 0$, and $\epsilon \in \mathbb{R}, \epsilon>0$, then

$$
T P_{(f-\epsilon e)}+e \leq \frac{1}{\epsilon^{2}} T\left(f^{2}\right)
$$

Proof: Let $f \in \mathcal{L}^{2}(T)$. As $P_{(f-\epsilon e)^{+}}$is the band projection onto the band generated by $(f-\epsilon e)^{+}$it follows that $P_{(f-\epsilon e)^{+}}(f-\epsilon e) \geq 0$ and thus

$$
P_{(f-\epsilon e)^{+}} f \geq \epsilon P_{(f-\epsilon e)^{+}} e \geq 0 .
$$

Band projections are dominated by the indentity map, so $P_{(f-\epsilon e)+} \leq I$, giving $|f| \geq$ $P_{(f-\epsilon e)^{+}}|f|$. From the positivity of band projections, $P_{(f-\epsilon e)^{+}}|f| \geq P_{(f-\epsilon e)^{+}} f$. Taking these observations together with (3.7) gives

$$
|f| \geq \epsilon P_{(f-\epsilon e)}+e \geq 0 .
$$

Multiplying (3.8) successively by $|f|, \epsilon P_{(f-\epsilon e)}+e \geq 0$ in the $f$-algebra $E^{u}$, universal completion of $E$, gives

$$
f^{2}=|f|^{2} \geq \epsilon|f| P_{(f-\epsilon e)}+e \geq\left(\epsilon P_{(f-\epsilon e)}+e\right)^{2} \geq 0 .
$$

The construction of the $f$-algebra structure on $E^{u}$ yields directly that $Q e \cdot Q e=Q e$ for all band projections $Q$. Hence

$$
\left(P_{(f-\epsilon e)}+e\right)^{2}=P_{(f-\epsilon e)}+P_{(f-\epsilon e)}+e^{2}=P_{(f-\epsilon e)}+e .
$$

Combining (3.9) and (3.10) gives

$$
f^{2} \geq \epsilon^{2} P_{(f-\epsilon))^{+}} e \geq 0 .
$$

Noting that $f^{2} \in E, T$ can be applied to (3.11) to give the desired inequality.

\section{Bienaymé Equality}

The Bienaymé equality of classical statistics gives that the variance of a finite sum of independent random variables coincides with variance of their sum. In this section we give a measure free conditional version of this result in $\mathcal{L}^{2}(T)$. Before we can proceed with this we require a result on $T$-conditionally independent random variables in $\mathcal{L}^{2}(T)$. 
Lemma 4.1 Let $E$ be a T-universally complete Riesz space with weak order unit, $e=$ $T e$, where $T$ is a strictly positive conditional expectation operator on $E$. Let $f, g \in \mathcal{L}^{2}(T)$. If $f$ and $g$ are $T$-conditionally independent then

$$
T f g=T f \cdot T g=T g \cdot T f .
$$

Proof: Let $T_{f}$ and $T_{g}$ denote the conditional expectations with ranges $\langle\mathcal{R}(T), f\rangle=E_{f}$ and $\langle\mathcal{R}(T), g\rangle=E_{g}$ respectively. Here $\langle\mathcal{R}(T), g\rangle$ denotes the order closed Riesz subspace of $E$ generated by $\mathcal{R}(T)$ and $g$, and similarly for $\langle\mathcal{R}(T), f\rangle$. The existence and uniqueness of $T_{f}$ and $T_{g}$ are given by the Radon-Nikodým Theorem, see [19]. Here $E_{g}$ and $E_{f}$ are $T$-conditionally independent as the $T$-conditional independence of $f$ and $g$ is defined in terms of the independence of $E_{f}$ and $E_{g}$, see Section 2. For each $h \in E, T_{f} h \in E_{f}$ and $T_{g} h \in E_{g}$. Now, as $E_{f}$ and $E_{g}$ are $T$-conditionally independent, from (2.5) with $E_{1}=E_{f}$ and $E_{2}=E_{g}$, we have

$$
T_{f}\left(T_{g} h\right)=T h=T_{g}\left(T_{f} h\right) .
$$

Applying (4.1) with $h=f g$ gives

$$
T(f g)=T_{f} T_{g}(f g)
$$

As $T_{f}$ and $T_{g}$ are averaging operators in $\mathcal{L}^{2}(T)$, by Corollary $3.3, T_{g}(f g)=g T_{g} f$. Thus

$$
T(f g)=T_{f} T_{g}(f g)=T_{f}\left(g T_{g} f\right),
$$

however taking (4.1) with $h=f$ yields $T_{g} f=T f$, which along with (4.2) gives

$$
T(f g)=T_{f}(g T f) .
$$

In (4.3), $T f \in \mathcal{R}(T) \subset E_{f}$, so by Corollary 3.3, $T_{f}(g T f)=T f \cdot T_{f} g$. Finally considering (4.1) with $h=g$ gives $T_{f} g=T g$. Thus

$$
T(f g)=T_{f}(g T f)=T f \cdot T_{f} g=T f \cdot T g .
$$

From Theorem 3.2, if $f \in \mathcal{L}^{2}(T)$ then $T f \in \mathcal{L}^{2}(T)$ which gives $(f-T f) \in \mathcal{L}^{2}(T)$. Hence, $(f-T f)^{2} \in \mathcal{L}^{1}(T)$ and so $T(f-T f)^{2}$ exists for all $f \in \mathcal{L}^{2}(T)$. We now define the variance of $f$ by

$$
\operatorname{var}(f)=T(f-T f)^{2}=T f^{2}-(T f)^{2} .
$$

Theorem 4.2 (Bienaymé Equality) Let E be a T-universally complete Riesz space with weak order unit, $e=T e$, where $T$ is a strictly positive conditional expectation operator on $E$. If $\left(f_{k}\right)_{k \in \mathbb{N}}$, is a T-conditionally independent sequence in $\mathcal{L}^{2}(T)$, then

$$
\operatorname{var}\left(\sum_{k=1}^{n} f_{k}\right)=\sum_{k=1}^{n} \operatorname{var}\left(f_{k}\right),
$$

for each $n \in \mathbb{N}$. 
Proof: As

$$
\left\langle f_{i_{1}}, \ldots f_{i_{j}}, \mathcal{R}(T)\right\rangle=\left\langle f_{i_{1}}-T f_{i_{1}}, \ldots f_{i_{j}}-T f_{i_{j}}, \mathcal{R}(T)\right\rangle,
$$

for each subset $\left\{i_{1}, \ldots, i_{j}\right\}$ of $\{1, \ldots, n\}$. Thus $f_{k}-T f_{k}, k=1,2, \ldots, n$ are $T$-conditionally independent. From Theorem [3.2, as $f_{i} \in \mathcal{L}^{2}(T)$ it follows that $T f_{i} \in \mathcal{L}^{2}(T)$ and consequently from Lemma 4.1 that

$$
T\left[\left(f_{i}-T f_{i}\right)\left(f_{j}-T f_{j}\right)\right]=\left[T\left(f_{i}-T f_{i}\right)\right] \cdot\left[T\left(f_{j}-T f_{j}\right)\right]
$$

for $i \neq j$. However, as $T$ is a projection, see Section 2,

$$
T\left(f_{k}-T f_{k}\right)=0, \quad \text { for each } k \in \mathbb{N},
$$

giving

$$
T\left[\left(f_{i}-T f_{i}\right)\left(f_{j}-T f_{j}\right)\right]=0,
$$

for $i \neq j$. From the definition of variance

$$
\operatorname{var}\left(\sum_{k=1}^{n} f_{k}\right)=T\left(\sum_{k=1}^{n} f_{k}-T \sum_{k=1}^{n} f_{k}\right)^{2}=T\left(\sum_{k=1}^{n}\left(f_{k}-T f_{k}\right)\right)^{2},
$$

which can be expanded to give

$$
\operatorname{var}\left(\sum_{k=1}^{n} f_{k}\right)=T \sum_{k=1}^{n}\left(f_{k}-T f_{k}\right)^{2}+T \sum_{j \neq k}\left(f_{j}-T f_{j}\right)\left(f_{k}-T f_{k}\right)
$$

Now applying (4.5) to (4.6) gives

$$
\operatorname{var}\left(\sum_{k=1}^{n} f_{k}\right)=\sum_{k=1}^{n} T\left(f_{k}-T f_{k}\right)^{2}=\sum_{k=1}^{n} \operatorname{var}\left(f_{k}\right) .
$$

\section{Bernoulli and Poisson Processes}

In classical probability, a Bernoulli process is one in which the events at any given time are independent of the events at all other times. The payoff of an event occuring is 1 unit and 0 units for it not occuring. Thus in the Riesz space setting, the process can be described by the sequence of independent band projections $P_{k}$ where $k$ indexes time and the payoff at time $k$ is $P_{k} e$. The probability of an event at time $k$ occuring must be independent of $k$, in the measure theoretic terms, this can be expressed as the expectation of each event is independent of time. This can be generalized to the conditional expectation of the events being time invariant, which lead to the Riesz space setting requirement that $T P_{k} e=f$, for all $k \in \mathbb{N}$. Here $T$ is some fixed conitional expectation operator. Thus we are led to the following formal definition of a Bernoulli process in Riesz spaces. 
Definition 5.1 Let $E$ be a Dedekind Riesz space with weak order unit, e, and conditional expectation operator $T$ with $T e=e$. Let $\left(P_{k}\right)_{k \in \mathbb{N}}$ be a sequence of $T$-conditionally independent band projections. We say that $\left(P_{k}\right)_{k \in \mathbb{N}}$ is a Bernoulli process if

$$
T P_{k} e=f \quad \text { for all } k \in \mathbb{N},
$$

for some fixed $f \in E$.

The payoff at time $n$ is thus

$$
S_{n}=\sum_{j=1}^{n} P_{j} e .
$$

We denote by $P_{S_{n}=j e}$ the band projection on the band where $S_{n}=j e$, in the notation used earlier $P_{S_{n}=j e}=\left(I-P_{\left(S_{n}-j e\right)^{+}}\right)\left(I-P_{\left(S_{n}-j e\right)^{-}}\right)$.

Theorem 5.2 Let $E$ be a T-universally complete Riesz space with weak order unit, $e=T e$, where $T$ is a strictly positive conditional expectation operator on $E$. Let $\left(P_{j}\right)_{j \in \mathbb{N}}$ be $T$-conditionally independent band projections with $T P_{j} e=f$ for all $j \in \mathbb{N}$ and $S_{n}=$ $\sum_{j=1}^{n} P_{j} e$. Then

$$
\begin{aligned}
T S_{n} & =n f \\
T P_{S_{n}=j e} e & =\frac{n !}{j !(n-j) !} f^{j}(e-f)^{n-j}, \\
\operatorname{var}\left(S_{n}\right) & =n f(e-f) .
\end{aligned}
$$

Proof: As $T P_{i} e=f$, (5.1) follows directly from applying $T$ to $S_{n}$.

Fix $n \in \mathbb{N}$ and let

$$
Q_{j}=\frac{1}{j !(n-j) !} \sum_{\sigma \in \Lambda} P_{k_{\sigma(1)}} \ldots P_{k_{\sigma(j)}}\left(I-P_{k_{\sigma(j+1)}}\right) \ldots\left(I-P_{k_{\sigma(n)}}\right) . \quad j=0, \ldots, n .
$$

Here $\Lambda$ denotes the set of all permutations of $\{1, \ldots, n\}$ and the division by $j !(n-j)$ ! is as there are $j !(n-j)$ ! permutations which yield the same band projection $P_{k_{\sigma(1)}} \ldots P_{k_{\sigma(j)}}(I-$ $\left.P_{k_{\sigma(j+1)}}\right) \ldots\left(I-P_{k_{\sigma(n)}}\right)$. Other permutations yield band projections disjoint from the above one. Thus $Q_{j}$ is a band projection, $Q_{0}, \ldots, Q_{n}$ partition the identity, $I$, in the sense that $Q_{i} Q_{j}=0$ for all $i \neq j$, and $\sum_{i=0}^{n} Q_{i}=I$. Moreover, from the definition of $Q_{j}$, it follows that $Q_{j} S_{n}=j Q_{j} e, j=0, \ldots, n$. Thus

$$
S_{n}=\sum_{j=0}^{n} Q_{j} S_{n}=\sum_{j=0}^{n} j Q_{j} e .
$$


The $T$-conditional independence of $P_{1}, \ldots, P_{n}$ and Lemma 4.1 applied iteratively give that

$$
\begin{aligned}
& T P_{k_{\sigma(1)}} \ldots P_{k_{\sigma(j)}}\left(I-P_{k_{\sigma(j+1)}}\right) \ldots\left(I-P_{k_{\sigma(n)}}\right) e \\
& =T\left(\left(P_{k_{\sigma(1)}} \ldots P_{k_{\sigma(j)}} e\right) \cdot\left(\left(I-P_{k_{\sigma(j+1)}}\right) \ldots\left(I-P_{k_{\sigma(n)}}\right) e\right)\right) \\
& =\left(T\left(P_{k_{\sigma(1)}} \ldots P_{k_{\sigma(j)}} e\right) \cdot\left(T\left(I-P_{k_{\sigma(j+1)}}\right) \ldots\left(I-P_{k_{\sigma(n)}}\right) e\right)\right) \\
& =\prod_{i=1}^{j} T P_{k \sigma(i)} e \cdot \prod_{i=J+1}^{n} T\left(I-P_{k_{\sigma(i)}}\right) e \\
& =f^{j}(e-f)^{n-j} .
\end{aligned}
$$

Hence

$$
T P_{S_{n}=j e} e=T Q_{j} e=\frac{1}{j !(n-j) !} \sum_{\sigma \in \Lambda} f^{j}(e-f)^{n-j},
$$

from which (5.2) follows as the cardinality of $\Lambda$ is $n$ !.

As $P_{1} e, \ldots, P_{n} e$ are $T$-conditionally independent and are in $\mathcal{L}^{2}(T)$, Bienaymé's equality applied to $S_{n}$ gives

$$
\operatorname{var}\left(S_{n}\right)=\sum_{k=1}^{n} \operatorname{var}\left(P_{k} e\right)
$$

From (4.4) applied to $P_{k} e$ we have

$$
\operatorname{var}\left(P_{k} e\right)=T P_{k} e-\left(T P_{k} e\right)^{2}=f-f^{2} .
$$

As $e$ is the multiplicative unit, combining (5.4) and (5.5) yields (5.3).

Theorem 5.3 (Bernoulli Law of Large Numbers) Let E be a T-universally complete Riesz space with weak order unit, $e=T e$, where $T$ is a strictly positive conditional expectation operator on $E$. Let $\left(P_{k}\right)_{k \in \mathbb{N}}$ be a Bernoulli process with partial sums $S_{n}$ and $T P_{k} e=f, k \in \mathbb{N}$. For each $\epsilon>0$,

$$
T P_{\left(\left|\frac{S_{n}}{n}-f\right|-\epsilon e\right)}+e \rightarrow 0
$$

as $n \rightarrow \infty$.

Proof: By the Tchebichev inequality,

$$
T P_{(|S-n f|-n \epsilon e)^{+}} e \leq \frac{1}{n^{2} \epsilon^{2}} T\left|S_{n}-n f\right|^{2} .
$$

However, from (4.4) and (5.1),

$$
T\left|S_{n}-n f\right|^{2}=\operatorname{var}\left(S_{n}\right) .
$$

Combining (5.6) with (5.7) and using (5.3) to simplify the result, gives

$$
T P_{(|S-n f|-n \epsilon e)^{+}} e \leq \frac{f(e-f)}{n \epsilon^{2}},
$$


from which the result follows upon observing that $P_{\left(\left|\frac{S_{n}}{n}-f\right|-\epsilon e\right)^{+}}=P_{(|S-n f|-n \epsilon e)^{+}}$.

One of the interesting features of Bernoulli's law of large numbers is that it gives not just the convergence of $T P_{\left(\left|\frac{S_{n}}{n}-f\right|-\epsilon e\right)^{+}}$to zero. It also gives some indication of the size of the band on which $\left|\frac{S_{n}}{n}-f\right|>\epsilon e$ by bounding the conditional expectation of the band projection applied to $e$ by $\frac{f(e-f)}{n \epsilon^{2}}$, hereby indicating an upper bound for the rate of convergence 'in probability'.

Using the results on martingale difference sequences developed for the study of mixingale in [12, Lemma 4.1] we obtain a weak law of large numbers for Bernoulli processes.

Theorem 5.4 (Weak law of large numbers) Let E be a T-universally complete Riesz space with weak order unit, $e=T e$, where $T$ is a strictly positive conditional expectation operator on E. Let $\left(P_{j}\right)_{j=1, \ldots, n}$ be T-conditionally independent band projections with $T P_{j} e=f$ for all $j=1, \ldots, n$ and $S_{n}=\sum_{j=1}^{n} P_{j} e$, then

$$
\lim _{n \rightarrow \infty} T\left|f-\frac{S_{n}}{n}\right|=0
$$

Proof: Setting $f_{i}=P_{i} e$ and $T_{i}$ to be the conditional expectation with range $\left\langle\mathcal{R}(T), P_{1} e, \ldots, P_{i} e\right\rangle$, it follows that $\left(g_{i}, T_{i}\right)$, where $g_{i}:=f_{i}-T_{i-1} f_{i}$, is a martingale difference sequence. Here $\left|f_{i}\right| \leq e$. Thus from [12, Lemma 4.1],

$$
\lim _{n \rightarrow \infty} T\left|\frac{1}{n} \sum_{i=1}^{n} g_{i}\right|=0 .
$$

The independence of the band projections $P_{i}, i \in \mathbb{N}$, gives that $T_{i-1} f_{i}=T f_{i}=f$. Hence (5.9) can be written as

$$
\lim _{n \rightarrow \infty} T\left|f-\frac{1}{n} \sum_{i=1}^{n} P_{i} e\right|=0,
$$

from which the theorem follows.

Before progressing further we need to define an exponential map on Riesz spaces.

Remark 5.5 Let $C([-1,1])$ denote the Riesz space of continuous real functions on $[-1,1]$. Set $f_{n}(t):=\left(1-\frac{t}{n}\right)^{n}$, then $f_{n} \in C([-1,1])$ and $f_{n}(t) \rightarrow e^{-t}=f(t)$ in order and the supremum norm on $C([-1,1])$. Thus, by [6, Theorem 3.1], for each $g \in E^{e}, f_{n}(g) \rightarrow f(g) e$-uniformly (and, thus, in order) as $n \rightarrow \infty$. In addition, by the functional calculus, $f(g)$ defines an element of $E^{e}$ which we will denote by $e^{-g}$. 
We now consider the sequences of Bernoulli processes known as Poisson sequences. Here the partial sums of each Bernoulli process form a Bernoulli process.

Theorem 5.6 (Poisson) Let E be a T-universally complete Riesz space with weak order unit, $e=T e$, where $T$ is a strictly positive conditional expectation operator on $E$. Let $P_{n, k}, k=1, \ldots, n, n \in \mathbb{N}$, be T-conditionally independent band projections with $T P_{n, k} e=g_{n}$ for all $k=1, \ldots, n, n \in \mathbb{N}$. If $S_{n}=\sum_{k=1}^{n} P_{n, k}$ e are $T$-conditionally independent with $T S_{n}=g, n \in \mathbb{N}$, then for each $j=0,1, \ldots$,

$$
T P_{S_{n}=j e} e \rightarrow \frac{g^{j}}{j !} e^{-g},
$$

e-uniformly as $n \rightarrow \infty$.

Proof: From (5.2),

$$
T P_{S_{n}=j e} e=\frac{n !}{j !(n-j) !} g_{n}^{j}\left(e-g_{n}\right)^{n-j},
$$

but (5.1) gives $g=T S_{n}=n g_{n}$. Hence

$$
T P_{S_{n}=j e} e=\frac{n !}{j !(n-j) !}\left(\frac{g}{n}\right)^{j}\left(e-\frac{g}{n}\right)^{n-j}
$$

which can be expanded to give

$$
\left(e-\frac{g}{n}\right)^{j} T P_{S_{n}=j e} e=\frac{g^{j}}{j !}\left(1-\frac{1}{n}\right)\left(1-\frac{2}{n}\right) \cdots\left(1-\frac{j-1}{n}\right)\left(e-\frac{g}{n}\right)^{n} .
$$

Taking the limit as $n \rightarrow \infty$ in (5.10) gives

$$
T P_{S_{n}=j e} e=\frac{g^{j}}{j !} \lim _{n \rightarrow \infty}\left(e-\frac{g}{n}\right)^{n},
$$

which together Remark 5.5 concludes the proof.

\section{References}

[1] C.D. Aliprantis, K. Border, Infinite dimensional analysis, 3rd Editiion, Springer Verlag, Berlin, 2006.

[2] K. Boulabiar, G. Buskes, A. Triki, Results in $f$-algebras, Positivity, Trends in Mathematics (2007), 73-96.

[3] G. Buskes, A. van Rooij, Almost $f$-algebras: Commutativity and the CuachySchwartz inequality, Positivity, 4 (2000), 227-231. 
[4] R. De MArr, A martingale convergence theorem in vector lattices, Canadian J. Math. 18 (1966), 424-432.

[5] P.G. Dodds, C.B. Huijsmans, B. De Pagter, Characterizations of conditional expectation-type operators, Pacific J. Math., 141 (1990), 55-77.

[6] J.J. Grobler, On the functional calculus in Archimedean Riesz spaces with applications to approximation theorems, Quaestiones Math. 11 (1988), 307 - 321.

[7] J.J. Grobler, Continuous stochastic processes in Riesz spaces: the Doob-meyer decomposition, Positivity 14 (2010), 731 - 751.

[8] J.J. Grobler, B. De PAGter, Operators representable as multiplicationconditional expectation operators, J. Operator Theory, 48 (2002), 15-40.

[9] C.C.A. Labuschagne, B.A. Watson, Discrete Stochastic Integration in Riesz Spaces, Positivity, 14, (2010), 859 - 575.

[10] W.-C. Kuo, C.C.A. Labuschagne, B.A. Watson, Discrete time stochastic processes on Riesz spaces, Indag. Math. N.S., 15 (2004), 435-451.

[11] W.-C. Kuo, C.C.A. Labuschagne, B.A. Watson, Conditional expectations on Riesz spaces, J. Math. Anal. Appl., 303 (2005), 509-521.

[12] W.-C. Kuo, J.J. Vardy, B.A. Watson, Mixingales on Riesz spaces, J. Math. Anal. Appl., 402 (2013), 731-738.

[13] M. LoÈve, Probability Theory I, 4th Edition, Springer Verlag, Berlin, 1977.

[14] W.A.J. Luxemburg, B. De Pagter, Representations of positive projections II, Positivity, 9 (2004), 569-605.

[15] H.H. Schaefer, Banach lattices and positive operators, Springer Verlag, Berlin, 1974.

[16] G. Stoica, Martingales in vector lattices, Bull. Math. Soc. Sci. Math. Roumanie. (N.S.), 34(82) (1990), 357-362.

[17] V. Troitsky, Martingales in Banach lattices, Positivity, 9 (2005), 437-456.

[18] J.J. Vardy, B.A. Watson, Markov processes in Riesz spaces, Positivity, 16 (2012), 373-391 and erratum on page 393.

[19] B. A. Watson, An Andô-Douglas type theorem in Riesz spaces with a conditional expectation, Positivity, 13 (2009), 543-558.

[20] A.C. ZaAnen, Introduction to Operator Theory in Riesz Space, Springer Verlag, Berlin, 1997. 